\title{
Hydrogenic impurity in a bilayer spherical quantum dot
}

\author{
V.I. Boichuk, I.V. Bilynskyi, R.Ya. Leshko \\ Ivan Franko Drohobych State Pedagogical University, Department of Theoretical Physics \\ 3, Stryiska str.,Drohobych 82100,Lviv region, Ukraine, e-mail: leshkoroman@mail.ru
}

\begin{abstract}
In the work, on the basis of the exact solution of the Poisson equation for a bilayer quantum dot with a positively charged donor ion in its centre, determined is the potential energy of interaction of this impurity ion with electron, taking into account different known values of $\mathrm{Si}$ and $\mathrm{SiO}_{2}$ dielectric permittivities. Using the found potential energy, the Schrödinger equation for the hydrogenic impurity in this system is solved exactly. The influence of external and internal radii on the electron spectrum is investigated. Described is the dependence of a squared matrix element for the dipole moment of interlevel transitions on the external and internal radii of the nanoheterosystem.
\end{abstract}

Keywords: hydrogenic impurity, Schrödinger equation, spherical quantum dot.

Manuscript received 16.12.08; accepted for publication 18.03.09; published online 20.03.09.

\section{Introduction}

Recently, a lot of attention has been given to impurity states in different confined systems, especially in quantum dots (QDs). A good deal of theoretical studies focus on monolayer spherical QDs in the continuum model approximation, which yields good agreement with experimental data. First theoretical researches of impurity states in QDs were reported in [1-5], where exact solutions of the Schrödinger equation with the Coulomb potential interaction between particles were obtained. By using exact solutions, the energy states of the donor located outside the spherical QD center were defined. Results show splitting and rearranging of levels depending on the QD radius, the donor position and the quantum well height. The reason of splitting and the rearrangement of levels is symmetry violation of the system and competition of the Coulomb interaction and the spatial confinement.

Modern experimental technologies and methods make it possible to create multi-layer spherically symmetric nanoheterosystems. For example, [6-7] describe the method of ion replacement under pressure by which $\beta-\mathrm{CdS} / \beta-\mathrm{HgS} / \mathrm{H}_{2} \mathrm{O}$ and the $\beta-\mathrm{CdS} / \beta-\mathrm{HgS} / \beta$ $\mathrm{CdS} / \mathrm{H}_{2} \mathrm{O}$ spherical nanostructures were made. In [8], for the spherical $\beta-\mathrm{HgS} / \beta-\mathrm{CdS} / \beta-\mathrm{HgS}$ nanoheterosystem the order of energy levels due to heterostructure layer dimensions was theoretically obtained and analyzed.

First attempts to study an impurity in a multilayer QD are described in [9], where the donor ground state was determined by the variation method. In [10], on the basis of the exact solution of the Schrödinger equation, the hydrogenic donor spectrum in a bilayer QD depending on the internal and external radii as well as heights of potential wells and potential barriers were determined. Also, the donor binding energy was determined.

However, these studies for the sake of simplicity of calculations used the approach of identical dielectric permeabilities of the media, which are equal to some average values. Such an approach has obvious drawbacks, especially for heterosystems with a large difference in dielectric permittivities. Report [5] takes account of the difference between the dielectric permittivities. But the chosen potential energy of the interaction between an impurity ion and an electron has a discontinuity on the heterosystem interface. The account of dielectric permittivities of the QD and matrix, and also polarising charges that arise at the separation boundaries of the heterosystem, can considerably change dimensions of an effective potential well [11].

In view of the above mentioned, in this work by using an exact solution of the Poisson equation for a bilayer QD with a positively charged impurity ion in its centre, the potential energy of interaction between the impurity ion and electron has been defined with account of different dielectric permittivity values of different QD layers and the matrix. The Schrödinger equation for a hydrogenic impurity in this system has been solved exactly using the found potential energy. The influence of the internal and external radii of the QD on the electron energy spectrum and on squared matrix element for the interlevel transition dipole moment has been determined. The specific calculations have been performed for the $\mathrm{Si} / \mathrm{SiO}_{2} / \mathrm{Si}$ spherical nanoheterostructure. 


\section{Theory}

2.1. The potential of a positively charged particle in the centre of a bilayer spherical $Q D$

We consider a three-layer spherical nanoheterosystem (bilayer QD). There is a positive charge $q$ in the centre of this system. The radius of the internal layer is $a$, and that of the external layer is $b$, therefore $a \leq b$. The dielectric permittivity of the internal layer of QD (region 1) is $\varepsilon_{1}$, that of the external layer of QD (region 2) is $\varepsilon_{2}$, while the dielectric permittivity of the matrix (region 3 ) is $\varepsilon_{3}$.

In order to determine the potential created by a charge $q$, it is necessary to solve the Poisson equation in these three regions. Due to spherical symmetry, it has the form

$$
\begin{array}{ll}
\frac{d^{2}}{d r^{2}} \Phi_{1}(r)+\frac{2}{r} \frac{d^{2}}{d r^{2}} \Phi_{1}(r)=-\frac{4 \pi q}{\varepsilon_{1}} \delta(r), & r \leq a, \\
\frac{d^{2}}{d r^{2}} \Phi_{2}(r)+\frac{2}{r} \frac{d}{d r} \Phi_{2}(r)=0, & a<r \leq b, \\
\frac{d^{2}}{d r^{2}} \Phi_{3}(r)+\frac{2}{r} \frac{d}{d r} \Phi_{3}(r)=0, & r>b .
\end{array}
$$

Corresponding solutions of these equations are written as

$$
\begin{array}{ll}
\Phi_{1}(r)=A_{1}+\frac{A_{2}}{r}, & r \leq a, \\
\Phi_{2}(r)=B_{1}+\frac{B_{2}}{r}, & a<r \leq b, \\
\Phi_{3}(r)=C_{1}+\frac{C_{2}}{r}, & r>b .
\end{array}
$$

Unknown coefficients are determined from the conditions of equality of the potential to zero at infinity, continuity of the potential and a normal component of the electric field vector at the separation boundary of the media and the Gauss theorem

$$
\begin{aligned}
& A_{1}=\frac{q\left(a \varepsilon_{1}\left(\varepsilon_{2}-\varepsilon_{3}\right)+b \varepsilon_{3}\left(\varepsilon_{1}-\varepsilon_{2}\right)\right)}{\varepsilon_{1} \varepsilon_{2} \varepsilon_{3} a b}, \quad A_{2}=\frac{q}{\varepsilon_{1}}, \quad C_{1}=0 \text {, } \\
& B_{1}=\frac{q\left(\varepsilon_{2}-\varepsilon_{3}\right)}{\varepsilon_{2} \varepsilon_{3} b}, \quad B_{2}=\frac{q}{\varepsilon_{2}}, \quad C_{2}=\frac{q}{\varepsilon_{3}} \text {. }
\end{aligned}
$$

By defining all the unknown coefficients, we found the potential created by the charge in the centre of QD.

\subsection{Hydrogenic impurity in the centre of a bilayer spherical $Q D$}

Let a positively charged ion of hydrogenic impurity $(q=e)$ be in the centre of the three-layer $\mathrm{Si} / \mathrm{SiO}_{2} / \mathrm{Si}$ spherical nanoheterostructure. The potential energy, due to the band mismatch in a quantum dot, is expressed as

$$
U(r)=\left\{\begin{array}{cc}
-U_{0}, & r \leq a, \\
0, & a<r \leq b, \quad U_{0}>0 . \\
-U_{0}, & r>b,
\end{array}\right.
$$

The potential energy of the interaction of an electron and impurity ion obtained by solving the Poisson equation (1) takes the form

$$
V(r)=e^{2}\left\{\begin{array}{lc}
-\frac{1}{\varepsilon_{1} r}-\frac{\varepsilon_{1}-\varepsilon_{2}}{\varepsilon_{1} \varepsilon_{2} a}-\frac{\varepsilon_{2}-\varepsilon_{1}}{\varepsilon_{1} \varepsilon_{2} b}, & r \leq a, \\
-\frac{1}{\varepsilon_{2} r}-\frac{\varepsilon_{2}-\varepsilon_{1}}{\varepsilon_{1} \varepsilon_{2} b}, & a<r \leq b, \\
-\frac{1}{\varepsilon_{1} r}, & r>b .
\end{array}\right.
$$

It is assumed that $\varepsilon_{3}=\varepsilon_{1}$.

The electron will induce polarization charges at the separation boundary of the media. The corresponding energy of electron - polarization charges interaction will be determined as in [11-14]

$W(r)=\left\{\begin{array}{lc}W_{1}(r), & r \leq a, \\ W_{2}(r), & a<r \leq b, \\ W_{3}(r), & r>b,\end{array}\right.$

$W_{1}(r)=\frac{e^{2}}{2 a \varepsilon_{1}}\left(\frac{\varepsilon_{1}-\varepsilon_{2}}{\varepsilon_{1}+\varepsilon_{2}}\right)\left\{\frac{a^{2}}{a^{2}-r^{2}}+\frac{\varepsilon_{1}}{\varepsilon_{2}} \times\right.$

$\left.\times_{2} F_{1}\left(1, \alpha_{1} ; \alpha_{1}+1 ;\left(\frac{r}{a}\right)^{2}\right)\right\}+\frac{e^{2}}{2 a} \frac{\varepsilon_{2}}{\left(\varepsilon_{1}-\varepsilon_{2}\right)} \sum_{n=0}^{\infty}\left(\frac{r}{a}\right)^{2 n} \times$,

$\times \frac{(2 n+1)^{2}}{\left(\varepsilon_{1} n+\varepsilon_{2}(n+1)\right) n} \frac{1}{Z_{n}}$,

$W_{2}(r)=\frac{e^{2}}{2 a \varepsilon_{2}}\left(\frac{\varepsilon_{1}-\varepsilon_{2}}{\varepsilon_{1}+\varepsilon_{2}}\right)\left\{\frac{a^{2}}{a^{2}-r^{2}}+\left(\frac{a}{r}\right)^{2} \times\right.$

$\left.\times{ }_{2} F_{1}\left(1, \alpha_{1} ; \alpha_{1}+1 ;\left(\frac{a}{r}\right)^{2}\right)\right\}+\frac{e^{2}}{2 b \varepsilon_{2}}\left(\frac{\varepsilon_{2}-\varepsilon_{1}}{\varepsilon_{2}+\varepsilon_{1}}\right) \times$

$\times\left\{\frac{b^{2}}{b^{2}-r^{2}}+\frac{\varepsilon_{2}}{\varepsilon_{1}}{ }_{2} F_{1}\left(1, \alpha_{2} ; \alpha_{2}+1 ;\left(\frac{r}{b}\right)^{2}\right)\right\}+$

$+\frac{e^{2}}{2 r \varepsilon_{2}} \sum_{n=0}^{\infty}\left\{\left(\frac{a}{r}\right)^{2 n+1} \frac{\left(\varepsilon_{1}-\varepsilon_{2}\right) n}{\left(\varepsilon_{1} n+\varepsilon_{2}(n+1)\right)}-\left(\frac{r}{b}\right)^{2 n+1} \times\right.$

$\left.\times \frac{\left(\varepsilon_{2}-\varepsilon_{1}\right)(n+1)}{\left(\varepsilon_{2} n+\varepsilon_{1}(n+1)\right)}-2\right\} \frac{1}{Z_{n}}$,

$W_{3}(r)=\frac{e^{2}}{2 b \varepsilon_{3}}\left(\frac{\varepsilon_{2}-\varepsilon_{1}}{\varepsilon_{2}+\varepsilon_{1}}\right)\left\{\frac{b^{2}}{b^{2}-r^{2}}+\left(\frac{b}{r}\right)^{2} \times\right.$

$\left.\times{ }_{2} F_{1}\left(1, \alpha_{2} ; \alpha_{2}+1 ;\left(\frac{b}{r}\right)^{2}\right)\right\}-\frac{e^{2}}{2 r} \frac{\varepsilon_{2}}{\left(\varepsilon_{2}-\varepsilon_{1}\right)} \times$

$\times \sum_{n=0}^{\infty}\left(\frac{b}{r}\right)^{2 n+1} \frac{(2 n+1)^{2}}{\left(\varepsilon_{2} n+\varepsilon_{1}(n+1)\right)(n+1)} \frac{1}{Z_{n}}$, 
where

$Z_{n}=1+\left(\frac{b}{a}\right)^{2 n+1} \frac{1}{n(n+1)} \frac{\left(\varepsilon_{1} n+\varepsilon_{2}(n+1)\right)\left(\varepsilon_{2} n+\varepsilon_{1}(n+1)\right)}{\left(\varepsilon_{1}-\varepsilon_{2}\right)\left(\varepsilon_{2}-\varepsilon_{1}\right)} ;$

$\alpha_{1}=\frac{\varepsilon_{2}}{\varepsilon_{1}+\varepsilon_{2}}, \alpha_{2}=\frac{\varepsilon_{1}}{\varepsilon_{2}+\varepsilon_{1}} ;$

${ }_{2} F_{1}(a, b, c ; x)$ is a generalized hypergeometric function.

The potential $W(r)$ is shown in Fig. 1 for two sets of QD sizes.

If we add expressions (4), (5) and (6), we will get the total electron potential energy in a bilayer QD

$$
\Pi(r)=\left\{\begin{array}{lc}
W_{1}(r)+\frac{-e^{2}}{\varepsilon_{1} r}-U_{1}^{*}(a, b), & r \leq a, \\
W_{2}(r)+\frac{-e^{2}}{\varepsilon_{2} r}-U_{2}^{*}(a), & a<r \leq b, \\
W_{3}(r)+\frac{-e^{2}}{\varepsilon_{1} r}-U_{0}, & r>b .
\end{array}\right.
$$

Here, the effective potential wells are introduced

$$
\begin{aligned}
& U_{1}^{*}(a, b)=\frac{e^{2}\left(\varepsilon_{1}-\varepsilon_{2}\right)}{\varepsilon_{1} \varepsilon_{2} a}+\frac{e^{2}\left(\varepsilon_{2}-\varepsilon_{1}\right)}{\varepsilon_{1} \varepsilon_{2} b}+U_{0}, \\
& U_{2}^{*}(b)=\frac{e^{2}\left(\varepsilon_{2}-\varepsilon_{1}\right)}{\varepsilon_{1} \varepsilon_{2} b} .
\end{aligned}
$$

It is seen from (8) that the effective potential wells depend on the sizes of heterosystem and dielectric permittivities. The operator of the total energy of the system is written as

$$
\mathbf{H}=-\frac{\hbar^{2}}{2} \nabla \frac{1}{m^{*}(r)} \nabla+\Pi(r),
$$

where

$$
m^{*}(r)=\left\{\begin{array}{lc}
m_{1}^{*}, & r \leq a, \\
m_{2}^{*}, & a<r \leq b, \\
m_{1}^{*}, & r>b .
\end{array}\right.
$$

The Schrödinger equation with Hamiltonian (9) is solved exactly, if we neglect the $W(r)$ term. The influence of that term will be taken into consideration in the first order of perturbation theory [11-14]. It is seen in Fig. 1, that potentials at the separation boundary of a heterosystem have different signs and forms, but during the integration opposite values "neutralize" each other, and the influence of $W(r)$ is small.

With regard for spherical symmetry of the problem, the wave function (it is the Schrödinger equation solution) can be represented in the form of a product of the radial and angular components

$\psi(r, \theta, \varphi)=R(r) \mathrm{Y}_{l}^{m}(\theta, \varphi)$,

where $\mathrm{Y}_{l}^{m}\left(\theta_{i}, \varphi_{i}\right)$ are spherical functions. Therefore, the radial Schrödinger equation can be written in three regions.

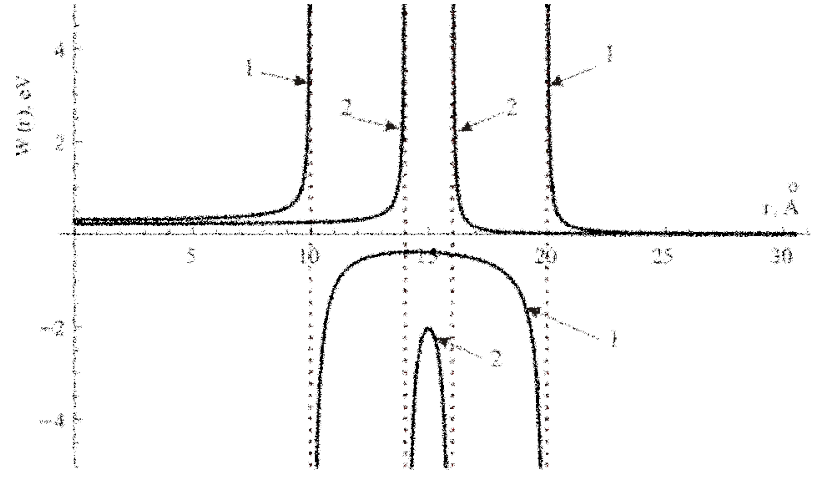

Fig. 1. The potential energy of electron-induced charges at the separation boundary $(1-a=10 \AA, b=20 \AA ; 2-a=14 \AA$, $b=16 \AA)$.

1. Region $r \leq a$. The radial Schrödinger equation has the form

$$
\begin{aligned}
& \left\{-\frac{\hbar^{2}}{2 m_{1}^{*}}\left(\frac{d^{2}}{d r^{2}}+\frac{2}{r} \frac{d}{d r}\right)+\frac{\hbar^{2} l(l+1)}{2 m_{1}^{*} r^{2}}-\right. \\
& \left.-\frac{e^{2}}{\varepsilon_{1} r}-U_{1}^{*}(a, b)-E\right\} R_{1}(r)=0 .
\end{aligned}
$$

In this region, there can be states with the energy both lower and higher than $-U_{1}^{*}(a, b)$. Therefore, the equation (12) is considered in these two energy ranges. Let $E<-U_{1}^{*}(a, b)$. If we introduce dimensionless values according to the expressions

$\xi=\alpha_{1 a} r, \alpha_{1 a}^{2}=-8 m_{1}^{*}\left(E+U_{1}^{*}(a, b)\right) / \hbar^{2}$,
$\lambda_{1}=2 m_{1}^{*} e^{2} /\left(\varepsilon_{1} \hbar^{2} \alpha_{1 a}\right), \quad R_{1}(\xi)=\xi^{-1} \rho_{1}(\xi)$

and perform some simple transformations, Eq. (12) is written

$\frac{\partial^{2} \rho_{1}(\xi)}{\partial \xi^{2}}+\left[-\frac{1}{4}+\frac{\lambda_{1}}{\xi}-\frac{l(l+1)}{\xi^{2}}\right] \rho_{1}(\xi)=0$.

We obtained the Whittaker equation that has two linearly independent solutions. If we take account of the wave function finiteness condition at the beginning of coordinates, the solution of Eq. (13) is represented by

$\rho_{1}(\xi)=C_{11} e^{-\frac{\xi}{2}} \xi^{l+1} F\left(l+1-\lambda_{1}, 2 l+2, \xi\right)$,

where $F(a, b ; x)$ is a confluent hypergeometric function of the first kind.

Let us consider another energy range: $-U_{1}^{*}(a, b)<E<-U_{0}$. We use the following notation $\xi=\alpha_{1 b} r ; \quad \alpha_{1 b}^{2}=2 m_{1}^{*}\left(E+U_{1}^{*}(a, b)\right) / \hbar^{2} ;$ $\beta_{1}=-m_{1}^{*} e^{2} /\left(\varepsilon_{1} \hbar^{2} \alpha_{1 b}\right) ; \quad R_{1}(\xi)=\xi^{-1} \rho_{1}(\xi)$. 
Equation (13) has the form

$$
\frac{\partial^{2} \rho_{1}(\xi)}{\partial \xi^{2}}+\left[1-\frac{2 \beta_{1}}{\xi}-\frac{l(l+1)}{\xi^{2}}\right] \rho_{1}(\xi)=0 \text {. }
$$

This is the Coulomb equation. The solution of this equation that satisfies the finiteness condition of the wave function can be represented by a hypergeometric function. Therefore, the solution of (15) in this energy range can be written as

$\rho_{1}(\xi)=C_{11} \frac{2^{l} e^{-\frac{\pi \beta_{1}}{2}}\left|\Gamma\left(l+1+i \beta_{1}\right)\right|}{\Gamma(2 l+2)} \times$

$\times e^{-i \xi_{\xi}^{l+1}} F\left(l+1-i \beta_{1}, 2 l+2,2 i \xi\right)$,

where $\Gamma(z)$ is the Euler gamma-function. equation

2. Region $\boldsymbol{a}<\boldsymbol{r} \leq \boldsymbol{b}$. The radial Schrödinger

$$
\begin{aligned}
& \left\{-\frac{\hbar^{2}}{2 m_{2}^{*}}\left(\frac{d^{2}}{d r^{2}}+\frac{2}{r} \frac{d}{d r}\right)+\frac{\hbar^{2} l(l+1)}{2 m_{2}^{*} r^{2}}-\right. \\
& \left.-\frac{e^{2}}{\varepsilon_{2} r}-U_{2}^{*}(b)-E\right\} R_{2}(r)=0,
\end{aligned}
$$

after introducing dimensionless variables and other parameters

$$
\begin{aligned}
& \xi=\alpha_{2} r, \alpha_{2}^{2}=-8 m_{2}^{*}\left(E+U_{2}^{*}(b)\right) / \hbar^{2}, \\
& \lambda_{2}=2 m_{2}^{*} e^{2} /\left(\varepsilon_{2} \hbar^{2} \alpha_{2}\right), \quad R_{2}(\xi)=\xi^{-1} \rho_{2}(\xi),
\end{aligned}
$$

is transformed into the Whittaker equation

$$
\frac{\partial^{2} \rho_{2}(\xi)}{\partial \xi^{2}}+\left[-\frac{1}{4}+\frac{\lambda_{2}}{\xi}-\frac{l(l+1)}{\xi^{2}}\right] \rho_{2}(\xi)=0 .
$$

Two linearly independent functions are a solution of this equation. Therefore,

$$
\begin{aligned}
& \rho_{2}(\xi)=C_{21} e^{-\frac{\xi}{2}} \xi^{l+1} F\left(l+1-\lambda_{2}, 2 l+2, \xi\right)+ \\
& +C_{22} e^{-\frac{\xi}{2}} \xi^{-l} \Phi\left(-l-\lambda_{2},-2 l, \xi\right),
\end{aligned}
$$

where $\Phi(a, b ; x)$ is a confluent hypergeometric function of the second kind.

3. Region $\boldsymbol{r}>\boldsymbol{b}$. The radial Schrödinger equation in this region is written in that form:

$$
\begin{aligned}
& \left\{-\frac{\hbar^{2}}{2 m_{1}^{*}}\left(\frac{d^{2}}{d r^{2}}+\frac{2}{r} \frac{d}{d r}\right)+\frac{\hbar^{2} l(l+1)}{2 m_{1}^{*} r^{2}}-\right. \\
& \left.-\frac{e^{2}}{\varepsilon_{1} r}-U_{0}-E\right\} R_{3}(r)=0 .
\end{aligned}
$$

By defining dimensionless values

$\xi=\alpha_{3} r, \alpha_{3}^{2}=-8 m_{1}^{*}\left(E+U_{0}\right) / \hbar^{2}$,

$\lambda_{3}=2 m_{1}^{*} e^{2} /\left(\varepsilon_{1} \hbar^{2} \alpha_{3}\right), R_{3}(\xi)=\xi^{-1} \rho_{3}(\xi)$,
Eq. (20) is transformed into the Whittaker equation

$\frac{\partial^{2} \rho_{3}(\xi)}{\partial \xi^{2}}+\left[-\frac{1}{4}+\frac{\lambda_{3}}{\xi}-\frac{l(l+1)}{\xi^{2}}\right] \rho_{3}(\xi)=0$.

Then the solution of (21) takes the form

$\rho_{3}(\xi)=C_{31} e^{-\frac{\xi}{2}} \xi^{-l} \Phi\left(-l-\lambda_{3},-2 l, \xi\right)$.

The function $\rho_{3}(\xi)$ can be written in the integral form

$\rho_{3}(\xi)=C_{31} \frac{e^{-\frac{\xi}{2}} \xi^{-l}}{\Gamma\left(-l-\lambda_{3}\right)} \int_{0}^{\infty} d t e^{-\xi t} t^{-l-\lambda_{3}-1}(1+t)^{-l+\lambda_{3}-1}$.

In the case of $E>-U_{2}^{*}(b)-\frac{e^{2}}{\varepsilon_{1} b}$, the energy spectrum becomes continuous, and in the case of $-U_{0}<E<-U_{2}^{*}(b)-\frac{e^{2}}{\varepsilon_{1} b}$ there exist quasi-stationary states.

We consider a discrete spectrum. The wave function and probability density flux are to be continuous at the separation boundary of the media. From those conditions and a normalization condition, one can define eigenvalues and eigenfunctions of a hydrogenic impurity in bilayer QD

$\left.R_{1}(r)\right|_{r=a}-\left.R_{2}(r)\right|_{r=a}=0$,

$\left.R_{2}(r)\right|_{r=b}-\left.R_{3}(r)\right|_{r=b}=0$,

$\left.\frac{1}{m_{1}^{*}} \frac{d}{d r} R_{1}(r)\right|_{r=a}-\left.\frac{1}{m_{2}^{*}} \frac{d}{d r} R_{2}(r)\right|_{r=a}=0$,

$\left.\frac{1}{m_{2}^{*}} \frac{d}{d r} R_{2}(r)\right|_{r=b}-\left.\frac{1}{m_{1}^{*}} \frac{d}{d r} R_{3}(r)\right|_{r=b}=0$,

$\int d \vec{r}|\psi(r, \theta, \varphi)|^{2}=1$.

The polarization term (6) can be taken into consideration in the first order of the perturbation theory [11-14]

$$
E_{\mathrm{pol}}=\int d \vec{r} \psi^{*}(\vec{r}) W(r) \psi(\vec{r}) .
$$

Thus, it is possible to define the total energy of the impurity electron.

\section{Analysis of the obtained results}

Above calculation of the electron discrete energy was performed for the heterostructure with the following parameters: $m_{1}^{*}=0.25, m_{2}^{*}=0.5, \varepsilon_{1}=11.7, \varepsilon_{2}=2.4$, $U_{0}=3.2 \mathrm{eV}$. Our solution are in good agreement with those in [10] provided instead of $\varepsilon_{1}$ and $\varepsilon_{2}$ in the derived formulae we substitute the average value of the dielectric permeability, because $U_{1}^{*}(a, b)=U_{0}$. The structure of solutions and quantum level order coincide with those calculated in previous works. However, account of the 
above-mentioned qualitatively influences the behavior of energy levels during the changing of the heterostructure sizes.

Fig. 2 shows the QD internal dependence of the donor ground state (at the external radius $b=180 \AA$ ). If we assume in calculations that the dielectric permeability of all heterostructure layers is identical under the condition $\varepsilon_{2}=\varepsilon_{1} \equiv \varepsilon_{\mathrm{SiO}_{2}}$, the radius dependence of the energy takes the form of the curve 1 . If we assume that $\varepsilon_{2}=\varepsilon_{1} \equiv \varepsilon_{\mathrm{Si}}$, then $E(a)$ is described by the curve 2 , but if we assume that $\varepsilon_{2}=\varepsilon_{1} \equiv\left(\varepsilon_{\mathrm{SiO}_{2}}+\varepsilon_{\mathrm{Si}}\right) / 2$, then the ground state energy as a function of the $a$ is represented by the curve 3. All these three functions are of the same structure. For a large QD radius, if $a \approx b$, the influence of $\mathrm{SiO}_{2}$ layer decreases, therefore the heterostructure energy levels approach the corresponding energies of a hydrogenic impurity in the bulk $\mathrm{Si}$ crystal, which are reduced by $U_{0}$ (the potential well depth). If $a_{0}<a<b$ $\left(25 \AA<a_{0}<50 \AA\right)$, then the energy of the system increases with the internal QD radius as caused by the spatial confinement. The farther reduction of the radius $\left(a<a_{0}\right)$ results in compensation of the growing kinetic energy by the potential energy of the electron attraction to an impurity ion. Hence, in this energy interval the energy does not practically depend on the internal QD radius.

With regard to various values of the dielectric permeability in heretostructure layers and using the exact solutions of the Poisson and Schrödinger equations, except (6), we obtained the function $E=E(a)$ that shows nonmonotonic character (curve 4). In the interval $a \approx b$, the energy is defined as $E=E_{\mathrm{Si}}$, therefore curves 2 and 4 coincide at large radii. With decreasing the QD internal radius, the electron energy is first decreased and then rises. The lowering of the energy level with decreased $a$ is caused by growth of the effective potential well $U_{1}^{*}(a, b)$, and the energy increase is caused by the spatial confinement. The further reduction of the QD radius is accompanied by hydrogen atom like behavior of the impurity in the bulk silicon crystal. In that region, there is no abrupt energy change, and curves 2 and 4 again approach each other. If we take into consideration the term (6), the function $E=E(a)$ is described by the curve 5 . As seen from the figure, the electron self-interaction potential (6) makes weak contribution into the total energy of the ground state. As an example, for $a=40 \AA b=180 \AA, E_{\mathrm{pol}}=0.07032 \mathrm{eV}$ and for $a=100 \AA, b=180 \AA, E_{\mathrm{pol}}=0.02813 \mathrm{eV}$. Thus, the term (6) will be neglected in further calculations.

In addition to the ground state, the excited ones are determined, too. Energy levels are set by the radial and orbital quantum numbers $n_{r}, l$ shown in Fig. 3 is the QD internal radius dependence of $E_{n_{r} l}$ in the case of a fixed external radius. It is seen that with the increase of the potential barrier thickness (decrease of the internal radius when the external one is fixed) to a certain value $(a \approx 30 \AA$, if $b \approx 200 \AA$ ) $2 s$ and $1 p$ energy states that is degenerated in free hydrogenic atom split.

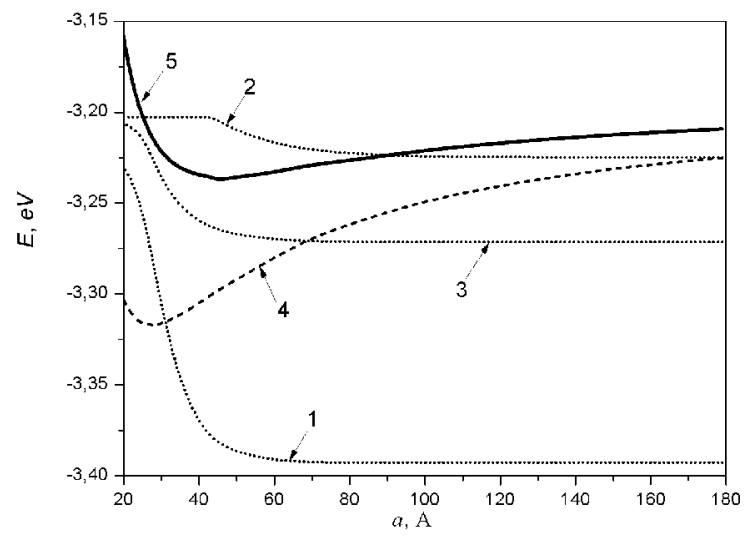

Fig. 2. The internal radius dependence of the donor ground state energy in bilayer QD. The external radius $b=180 \AA$.

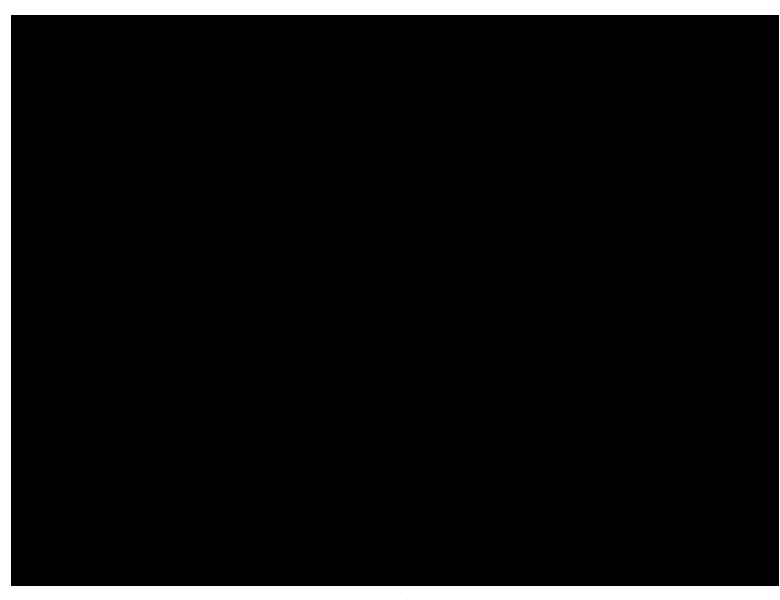

$a$
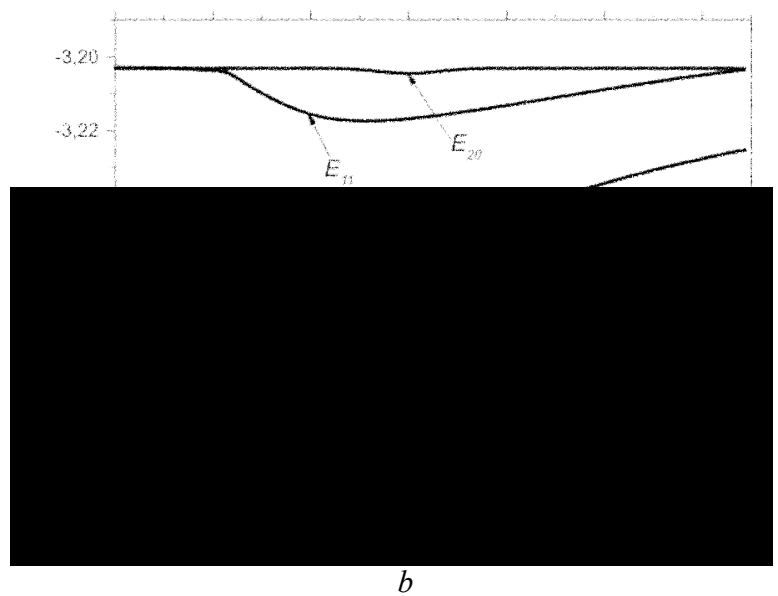

Fig. 3. The internal radius dependence of the hydrogenic impurity energy in bilayer QD. The external radius $b=200 \AA$ (left), $b=150 \AA$ (right).

In Fig. 4, the dependence of $E_{n_{r} l}$ on the external radius is shown, when the internal radius is fixed $(a=80 \AA)$. As seen, the external QD radius $b$ reduction results in the system energy increase. It is caused by the $U_{1}^{*}(a, b)$ effective potential well depth reduction and the $U_{2}^{*}(b)$ barrier height rise. 


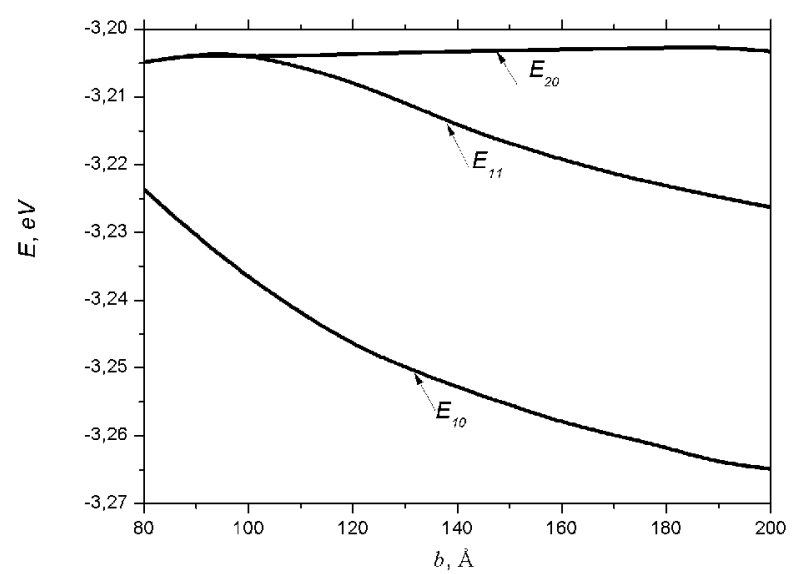

Fig. 4. The external radius dependence of the energy levels of the hydrogenic impurity.

Studying the physical processes caused by absorption and radiation of light, it is important to define the dependence of a matrix element square of the dipole moment for electron interlevel transitions. In this paper, we defined dipole moments over the function corresponding to $1 s\left(\psi_{10}\right)$ and $1 p\left(\psi_{11}\right)$ states:

$$
\begin{aligned}
& \left|\mathbf{P}_{10,11}\right|^{2}=e^{2}\left|\mathbf{D}_{10,11}\right|^{2}, \\
& \mathbf{D}_{10,11}=\int d \mathbf{r} \psi_{10}(\mathbf{r}) \mathbf{r} \psi_{11}(\mathbf{r}) .
\end{aligned}
$$

Fig. 5 shows plots of the $\left|\mathbf{P}_{10,11}\right|^{2} /\left|\mathbf{P}_{10,11}^{0}\right|^{2}$ dependence on the internal radius, where $\left|\mathbf{P}_{10,11}^{0}\right|^{2}$ is the square of a matrix element of a dipole moment for interlevel transitions, which is based on the respective functions of the hydrogenic impurity in the bulk silicon crystal. It is seen that the increase of $a$ leads to the rise of $\left|\mathbf{P}_{10,11}\right|^{2}$. There is an interval of $a$, which depends on $b$, where $\left|\mathbf{P}_{10,11}\right|^{2} /\left|\mathbf{P}_{10,11}^{0}\right|^{2}>1$. With the further increase of $a$ (decrease of the $\mathrm{SiO}_{2}$ layer), the value of $\left|\mathbf{P}_{10,11}\right|^{2}$ approaches to the respective value in the bulk crystal, i.e. $\left|\mathbf{P}_{10,11}\right|^{2} /\left|\mathbf{P}_{10,11}^{0}\right|^{2} \rightarrow 1$. In the case of a very large DQ radius, we actually get a single layer QD. The behavior of the matrix element square of a dipole moment for interlevel transitions in this case is shown in Fig. 5b. It is seen from Fig. 5b that the increase of the quasimonolayer QD radius causes the rise of $\left|\mathbf{P}_{10,11}\right|^{2} /\left|\mathbf{P}_{10,11}^{0}\right|^{2}$, and the further increase of $a$ (transition to the bulk crystal) leads to a monotonic change of the matrix element square of a dipole moment for interlevel transitions. Note that $\left|\mathbf{P}_{10,11}\right|^{2} /\left|\mathbf{P}_{10,11}^{0}\right|^{2} \rightarrow 1$.
Fig. 6 illustrates the dependence on the external QD radius for the matrix element square of a dipole moment for interlevel transitions in the case of a fixed internal radius. If $b \rightarrow a,\left|\mathbf{P}_{10,11}\right|^{2} /\left|\mathbf{P}_{10,11}^{0}\right|^{2} \rightarrow 1$, as the $\mathrm{SiO}_{2}$ layer thickness vanishes. The increase of $b$ leads first to the rise of $\left|\mathbf{P}_{10,11}\right|^{2} /\left|\mathbf{P}_{10,11}^{0}\right|^{2}$, and then to smooth change. This behavior testifies to the decrease in matrix influence on the heterosystem properties.
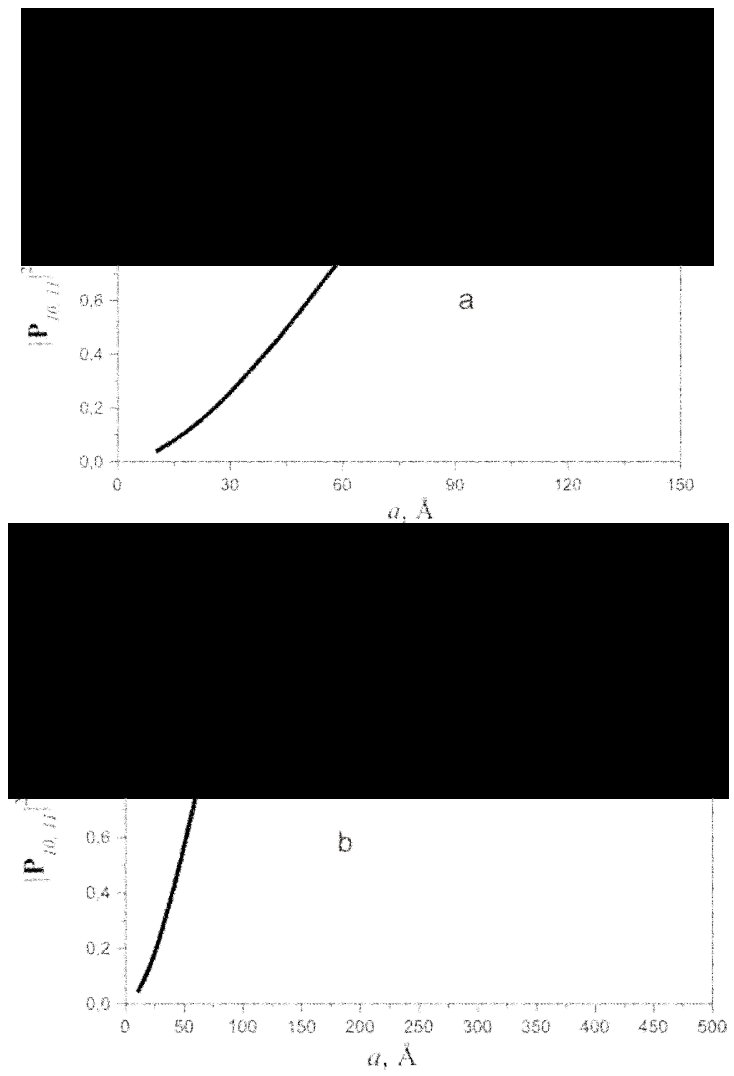

Fig. 5. The dependence of the matrix element square of a dipole momentum for interlevel transitions on $a: b=150 \AA$ (a), $b=500 \AA(\mathrm{b})$.

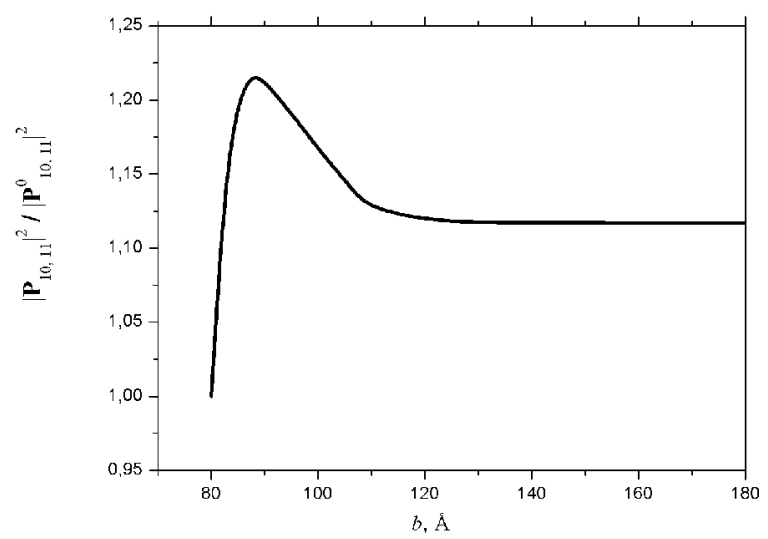

Fig. 6. The external QD radius dependence of the matrix element square of a dipole momentum for interlevel transitions (internal radius $a=80 \AA$ ). 
Therefore, we obtained an exact solution of the Poisson equation for a positively charged impurity ion in the centre of bilayer QD. It allowed us to derive the total electron potential energy by means of effective potential wells for an electron in the heterostructure. Using the found potential energy, the Schrödinger equation is solved precisely for discrete spectrum in the three-layer spherical QD. We also obtained the dependence of the matrix element square of an interlevel transitions dipole momentum based on the function of $1 s$ and $1 p$ states on nanostructure dimensions.

\section{References}

1. Jia-Lin Zhu, Exact solution for hydrogenic donor states in a spherically rectangular quantum well // Phys. Rev. B 39(12), p. 8781-8783 (1989).

2. Jia-Lin Zhu, Jia-Jiong Xiong, Bing-Lin Gu, Confined electron and hydrogenic donor states in a spherical quantum dot of GaAs- $\mathrm{Ga}_{1-\mathrm{x}} \mathrm{Al}_{\mathrm{x}} \mathrm{As} / /$ Phys. Rev. B 41(9), p. 6001-6007 (1990).

3. Jia-Lin Zhu, Xi Chen, Spectrum and binding of an off-center donor in a spherical quantum dot // Phys. Rev. B 50(7), p. 4497-4502 (1994).

4. Chun-Ching Yang, Li-Chi Liu, and Shih-Hsin Chang, Eigenstates and fine structure of a hydrogenic impurity in a spherical quantum dot // Phys. Rev. B 58(4), p. 1954-1961 (1998).

5. M.V. Tkach, V.A. Golovatskyi, Ya.M. Berezovskyi, Spectrum and wave functions of hydrogenic impurity placed in the center of a quantum dot // Fizika $i$ khimiya tverdogo tila 4(2), p. 213-220 (2003) (in Ukrainian).

6. D. Schooss, A. Mews, A. Eychmuller, H. Weller, Quantum-dot quantum well CdS/HgS/CdS: Theory and experiment // Phys. Rev. B 49(24), p. 1707217078 (1994).
7. A. Mews, A.V. Kadavanich, U. Banin, A.P. Alivasatos, Structural and spectroscopic investigations of $\mathrm{CdS} / \mathrm{HgS} / \mathrm{CdS}$ quantum-dot quantum wells // Phys. Rev. B 53(20), p. R13242-R 13245 (1996).

8. N.V. Tkach, V.A. Golovatskyi, Quasi-stationary states of an electron in a spherical nanoheterosystem $\beta-\mathrm{HgS} / \beta-\mathrm{CdS} / \beta-\mathrm{HgS} / /$ Fizika tverdogo tela 41(11), p. 2081-2083 (1999) (in Russian).

9. S. Chaudhuri, Hydrogenic-impurity ground state in GaAs- $\mathrm{Ga}_{1-\mathrm{x}} \mathrm{Al}_{\mathrm{x}} \mathrm{As}$ multiple-quantum-well structures // Phys. Rev. B 28(8), p. 4480-4488 (1983).

10. Cheng-Ying Hsieh, Lower lying states of hydrogenic impurity in a multi-layer quantum dot // Chinese J. Phys. 38(3), p. 478-490 (2000).

11. V.I. Boichuk, R.Yu. Kubai, G.M. Godovanets, I.S. Shevchuk, Investigation of polarization influence on the energy of an electron or hole in a semiconductor nanoheterosystem (as based on structures $\left.\quad \mathrm{Si} / \mathrm{SiO}_{2}, \quad \beta-\mathrm{HgS} / \mathrm{CdS}\right) \quad / / \quad$ Zhurnal fizychnykh doslidzhen' 10(3), p. 220-226 (2006) (in Ukrainian).

12. L.V. Keldysh, O.V. Konstantinov, V.I. Perel', Polarization effects accompanying interband light absorption in semiconductors with applied strong electric field // Fizika tekhnika poluprovodnikov 3, p. 1042 (1969) (in Russian).

13. N.A. Yefremov, S.I. Pokutnyi, Macroscopic local charge states in ultradisperse media // Fizika tverdogo tela 27(1) p. 48-56 (1985) (in Russian).

14. V.I. Boichuk, R.Yu. Kubai, I.V. Bilyns'kyi, Influence of image forces on the electron energy spectrum in complex spherical microcrystal $\mathrm{CdS} / \beta$ $\mathrm{HgS} / \mathrm{H}_{2} \mathrm{O} / /$ Zhurnal fizychnykh doslidzhen' 3(2), p. 187-191 (1999) (in Ukrainian). 